\title{
Trabalho em enfermagem: análise da tendência dos salários no Brasil
}

\author{
Nursing work: analysis of wage trends in Brazil \\ Trabajo de enfermería: análisis de las tendencias salariales en Brasil
}

Luiza Jane Eyre de Souza Vieira ORCID: https://orcid.org/0000-0002-5220-027X Universidade de Fortaleza, Brasil E-mail: janeeyre@unifor.br

Raimunda Magalhães da Silva ORCID: https://orcid.org/0000-0001-5353-7520 Universidade de Fortaleza, Brasil E-mail: rmsilva@unifor.br Carlos Garcia Filho ORCID: https://orcid.org/0000-0002-0345-6033 Universidade de Fortaleza, Brasil E-mail: cgarciafilho@gmail.com

Indara Cavalcante Bezerra

ORCID: https://orcid.org/0000-0003-0647-2490 Universidade estadual do Ceará, Brasil

E-mail: indaracavalcante@yahoo.com.br Antonio Rodrigues Ferreira Júnior ORCID: https://orcid.org/0000-0002-9483-8060 Universidade Estadual do Ceará, Brasil E-mail: arodrigues.junior@uece.br

José Maria Ximenes Guimarães ORCID: https://orcid.org/0000-0002-5682-6106 Universidade Estadual do Ceará, Brasil E-mail: jose.ximenes@uece.br

\section{Resumo}

Objetivou-se analisar a tendência da remuneração dos profissionais de enfermagem no Brasil. Trata-se de estudo transversal que utilizou dados secundários obtidos da Relação Anual de Informações Sociais (RAIS) que compila informações da atividade trabalhista no País, possibilitando a identificação da diferença entre a remuneração de profissionais de enfermagem de níveis superior e médio, nos anos de 2003 e 2018. Analisaram-se as variáveis Classificação Brasileira de Ocupações-CBO 2002, horas contratadas e valor da remuneração média nominal, corrigindo-se valores financeiros pelo Índice de Preços ao Consumidor Amplo. Evidenciou-se que a remuneração de enfermeiros e técnicos aproximando-se nos últimos 15 anos; há discreta estagnação do valor recebido pelos enfermeiros; aumento real do valor recebido pelos técnicos, que acompanhou o salário mínimo. A composição dos trabalhadores com carga horária semanal de 40 a 44 horas modificou-se; ocorreu expansão de 94,9\% na quantidade de vínculos entre 2003 (350.470) e 2018 (682.532) e modificação na proporção entre grupos. Em 2003, tinha-se 84,4\% de técnicos e 15,6\% de enfermeiros; em 2018, 71,4\% técnicos e 28,6\% enfermeiros. Os resultados evidenciam tendência de aproximação entre a remuneração salarial da equipe de enfermagem, diminuição relativa no número de técnicos e aumento relativo no número de enfermeiros.

Palavras-chave: Enfermagem; Economia da enfermagem; Assistentes de enfermagem; Enfermeiro; Salários e benefícios.

\footnotetext{
Abstract

The scope of the study was to analyzes the trends in the remuneration of the work of nursing professionals, including those with higher and secondary education. This is a cross-sectional study that used data from the Annual List of Social Information (Relação Anual de Informações Sociais - RAIS), which compiles information on labor activity in the country, thus enabling the identification of the difference between the remunerations of nursing professionals with higher education and those with secondary education in the years 2003 and 2018 in Brazil. The variables 2002 Brazilian Classification of Occupations (Classificação Brasileira de Ocupações - CBO 2002), number of hours hired and the value of the average nominal remuneration were analyzed and the financial values were corrected by the Broad Consumer Price Index. The remuneration of nurses and technicians over the past 15 years. There is a slight stagnation in the amount received by nurses and a real increase in the amount received by technicians, which accompanied the minimum wage. The composition of workers with a weekly workload of 40 to 44 hours has changed. There was an expansion of $94.9 \%$ in the number of bonds between $2003(350,470)$ and $2018(682,532)$ and a change
} 
in the proportion between groups. In 2003, $84.4 \%$ were technicians and $15.6 \%$ were nurses. In $2018,71.4 \%$ were technicians and $28.6 \%$ were nurses. There is a trend towards an approximation between remunerations of the nursing staff and a relative decrease in the number of technicians and a relative increase in the number of nurses.

Keywords: Nursing; Nursing economics; Nursing assistants; Nurse; Salaries and fringe benefits.

\section{Resumen}

El objetivo de este estudio fue analizar la tendencia de la remuneración de los profesionales de enfermería en Brasil. Estudio transversal que utilizó datos secundarios obtenidos del Relación Anual de Información Social (RAIS) que recopila información sobre la actividad laboral en el país, permitiendo identificar la diferencia entre la remuneración de los profesionales de enfermería con educación superior y secundaria, en los años 2003 y 2018. Se analizaron las variables Clasificación Brasileña de Ocupaciones-CBO 2002, horas contratadas y el valor de la remuneración nominal promedio, corrigiendo valores financieros por el Índice de Precios al Consumidor Amplio. La remuneración de enfermeros y técnicos se ha venido acercando en los últimos 15 años; hay un leve estancamiento en la cantidad recibida por enfermeros; Incremento real de la cantidad percibida por los técnicos, que acompañó al salario mínimo. La composición de los trabajadores con una carga de trabajo semanal de 40 a 44 horas ha cambiado; hubo una expansión del 94,9\% en el número de bonos entre $2003(350,470)$ y $2018(682,532)$ y un cambio en la proporción entre grupos. En 2003 había 84,4\% de técnicos y 15,6\% de enfermeros; en 2018, 71,4\% técnicos y 28,6\% enfermeros. Los resultados muestran una tendencia de aproximación entre la remuneración salarial del equipo de enfermería, una disminución relativa en el número de técnicos y un aumento relativo en el número de enfermeros.

Palabras clave: Enfermería; Economía de la enfermería; Auxiliares de enfermería; Enfermero; Salarios y beneficios.

\section{Introdução}

O mercado de trabalho em saúde e em enfermagem constitui temática relevante nos debates nacional e internacional, com análises pautadas na economia e na sociologia das profissões, em que se tomam como objeto as tendências de mercado público e/ou privado - a regulação e as relações entre formação e mundo do trabalho. Nesse contexto, observa-se centralidade nos processos de globalização e de implementação de políticas econômicas de cunho neoliberal, os quais produzem, em alguma medida, impactos no trabalho em saúde.

Destarte, as alterações ocorridas na economia mundial, desde a década de 1970, afetaram o mercado de trabalho. Assim, os processos de reestruturação produtiva desencadearam a redefinição da relação capital e trabalho, ocasionado enfraquecimento da classe trabalhadora. Com efeito, foram disseminadas, no Brasil, novas formas de contratação, flexibilização dos direitos trabalhistas, ampliação da jornada de trabalho e das situações de insalubridade e periculosidade no trabalho, culminando na precarização do trabalho que coloca o trabalhador em condições de submissão ante a superexploração, como condição de manutenção do emprego (Melo et al., 2016).

No Brasil, particularmente, os profissionais de enfermagem representam a maior força de trabalho no sistema de saúde. Contudo, ainda se observam desafios no âmbito do exercício profissional da enfermagem, expressos na qualificação profissional, inadequações entre a formação e as exigências do mercado, carga excessiva de trabalho, conflitos entre equipes multiprofissionais, não valorização e reconhecimento social (Spetz, 2016). Desse modo, os profissionais de Enfermagem brasileiros, nos últimos 20 anos, têm experimentado mudanças importantes no mercado de trabalho, que tem demonstrado fragilidades relacionadas ao assalariamento com diferentes formas de vínculos trabalhistas, como pagamentos por plantões ou por hora trabalhada, contratos curtos e temporários (Machado et al., 2019).

Segundo dados da Pesquisa Perfil da Enfermagem no Brasil (PPEB), do Conselho Federal de Enfermagem, em 2019, o quantitativo de profissionais da Enfermagem no Brasil, perfez 2.169.402 profissionais, sendo 527.842 enfermeiros e 1.641.560 profissionais de nível médio, dos quais 1.230.182 são técnicos e 411.378 são auxiliares de enfermagem (Conselho Federal de Enfermagem [COFEN], 2019). Quantidade significativa desses trabalhadores, a maioria de nível médio, vive com baixos rendimentos salariais, precarização das condições de trabalho, jornadas múltiplas de trabalho e multiempregos (Machado et al., 2019; Silva et al., 2020). 
Ao discorrer sobre as tendências estruturais do mundo do trabalho, Pochmann (2020) alerta que as relações laborais não se apresentam lineares com o decorrer das décadas. Essas tendem a sofrer impactos das trajetórias dos sistemas produtivos e dos formatos que regulam o funcionamento do mercado de trabalho.

Com efeito, comparar a variação dos rendimentos salariais de técnicos e auxiliares de enfermagem com os rendimentos dos enfermeiros, pode subsidiar novas estratégias e políticas sobre como enfrentar a precarização e garantir segurança e melhores condições de trabalho, assim como remunerações mais equitativas entre a categoria de enfermagem. Ademais, entende-se que este estudo pode fomentar debates que confrontem o avanço de políticas neoliberais e interveniência direta na vida laboral dessa categoria.

Amplia-se a relevância na medida em que ainda é escassa na literatura a disponibilidade de artigos sobre salários, perda do poder aquisitivo, instabilidade laboral, modalidades contratuais que se avolumam e interferem, sobremaneira, na oferta de uma atenção qualificada (Ariste \& Béjaoui, 2015; Guerrero \& Pulido, 2010) e na qualidade de vida desses profissionais.

Alinhado com este raciocínio, tem-se como objetivo analisar a tendência da remuneração do trabalho de profissionais de enfermagem, incluindo os de níveis superior e médio.

\section{Metodologia}

Trata-se de um estudo quantitativo, com desenho transversal. Ressalta-se que estudos de natureza quantitativa são adequados para a coleta e análise de dados mensuráveis, por meio da aplicação de matemáticas, como a estatística (Pereira et al., 2018). Foram utilizados dados secundários obtidos da Relação Anual de Informações Sociais (RAIS), que é um registro administrativo nacional e obrigatório para todos os estabelecimentos, inclusive os sem ocorrência de vínculo empregatício, para analisar a diferença entre a remuneração de profissionais de enfermagem de níveis superior e médio entre os anos de 2003 e 2018 no Brasil.

A RAIS é elaborada pelo Ministério da Economia (ME) e tem por objetivo prover informações para o controle da atividade trabalhista no País, subsidiar a elaboração de estatísticas do trabalho e disponibilizar informações do mercado de trabalho às entidades governamentais. As bases de dados da RAIS são geradas com base na análise das declarações originais preenchidas pelos estabelecimentos. Após organização e checagem dos dados, os bancos de dados são disponibilizados para acesso público. Quanto à unidade de análise, as informações dos empregados são prestadas para cada vínculo de emprego, portanto um trabalhador pode constar na RAIS mais de uma vez, de acordo com a quantidade de vínculos que possui no ano da coleta.

Após download dos bancos de dados de cada um dos Estados brasileiros para os anos abordados neste estudo, disponível no site do ME, foram selecionadas as variáveis de interesse, Classificação Brasileira de Ocupações - CBO 2002, Quantidade de Horas Contratadas e Valor da Remuneração Média Nominal, e constituído um banco único. Os valores financeiros foram corrigidos pelo Índice de Preços ao Consumidor Amplo (IPCA) e os outliers foram excluídos. Considerou-se como outlier o valor de remuneração superior a $\mathrm{m}+$ ou $\pm 6 \mathrm{dp}(\mathrm{m}=$ média; $\mathrm{dp}=$ desvio padrão). Somente foram analisados os dados referentes à carga horária de 40 a 44 horas semanais. Os CBO selecionados foram "3222 - Técnicos e auxiliares de enfermagem" e "2235 - Enfermeiros e afins". Neste artigo, o termo técnico foi utilizado como sinônimo do primeiro e enfermeiro do segundo. O termo enfermagem foi utilizado como sinônimo para a soma dos dois grupos.

Inicialmente, foram calculadas as estatísticas descritivas por grupo (nível médio $x$ nível superior) para avaliar a heterogeneidade dos valores. Considerou-se como renda média o intervalo entre $2 / 3 *$ me e $2 *$ me (onde me $=$ mediana). Nessa investigação, os parâmetros de renda utilizados pelo Pew Research Center (2015) para definir classe média foram adaptados 
para descrever a "classe média" de enfermeiros e técnicos de enfermagem. Essa escolha deu-se pela facilidade de cálculo e interpretação dos resultados, possibilitando comparações em pesquisas futuras.

Para analisar a desigualdade de renda observada e sua evolução utilizou-se a decomposição dos índices de Theil por grupos. Essa técnica possibilita mensurar a desigualdade de renda de cada um dos grupos, bem como entre os grupos (Haughton, 2009). Foram utilizados os dois indicadores de desigualdade de Theil, o L de Theil e o T de Theil. Seu cálculo foi realizado por meio do pacote do IC2 (Inequality and Concentration Indices and Curves) da linguagem R, que também foi utilizada por meio do software R-Studio versão 1.1.456, para manusear os bancos de dados da RAIS e elaborar os gráficos.

\section{Resultados}

A Tabela 1 apresenta as estatísticas descritivas dos rendimentos para enfermeiros e técnicos de enfermagem corrigidos pelo IPCA para preços de dezembro de 2018.

Tabela 1 - Estatísticas descritivas do rendimento real a preços constantes de 2018 de técnicos e enfermeiros, por anos selecionados (2003-2018).

\begin{tabular}{ccccc}
\hline \multirow{2}{*}{ Ano } & Grupo & Média & Mediana & Desvio Padrão \\
\hline \multirow{2}{*}{2003} & Técnico & 1753.07 & 1442.56 & 1127.50 \\
& Enfermeiro & 4522.51 & 4135.53 & 2353.83 \\
\hline \multirow{2}{*}{2008} & Técnico & 1993.89 & 1556.11 & 1367.39 \\
& Enfermeiro & 4762.19 & 4349.70 & 2489.07 \\
\hline \multirow{2}{*}{2013} & Técnico & 2343.65 & 1814.25 & 1610.13 \\
& Enfermeiro & 5119.62 & 4297.64 & 2988.71 \\
\hline \multirow{2}{*}{2018} & Técnico & 2360.35 & 1901.54 & 1432.03 \\
& Enfermeiro & 4940.25 & 4099.09 & 2841.50 \\
\hline
\end{tabular}

Fonte: Autores, com base nos dados da RAIS.

As estatísticas descritivas mostram aumento da média $(34,6 \%)$ e da mediana $(31,8 \%)$ da remuneração para técnicos de enfermagem no período estudado. O ritmo do aumento não é constante, concentra-se no intervalo de 2003 a 2013 (33,7\% para média e 25,8\% para mediana) e reduz sua intensidade entre 2013 a 2018 (0,7\% para média e 4,8\% para mediana).

No caso dos enfermeiros, ocorre aumento da média (13,2\%) entre 2003 e 2013 e há redução $(-3,5 \%)$ nesse parâmetro para o último período estudado. Considerando os 15 anos abordados nesta pesquisa, há elevação da média $(9,2 \%)$.

Por outro lado, ao considerar-se a mediana, a situação é diferente, ocorre estabilidade desse parâmetro durante o período estudado, de fato, uma pequena redução $(-0,01 \%)$. Observou-se aumento da mediana (5,2\%) no período de 2003 a 2008 e redução $(-5,8 \%)$ entre 2008 a 2018.

Dadas as características dessas medidas de tendência central, pode-se explicar essa divergência entre aumento da média e diminuição da mediana da remuneração recorrendo-se a mudanças na distribuição dos dados e as características desses parâmetros, dado que a média é mais sensível a valores extremos do que a mediana. O aumento do desvio-padrão para o período estudado reforça essa hipótese.

Um aspecto alternativo para analisar a evolução da remuneração do trabalho é substituir valores pontuais por intervalos. Os resultados da Tabela 2 mostram expressivo aumento (31,82\%) dos rendimentos da "classe média" dos técnicos de enfermagem para o período estudado. Por outro lado, o rendimento da "classe média" dos enfermeiros apresentou discreta 
redução $(-0,88 \%)$ no período. Essa diferença, na evolução dos valores, levou a um incremento da sobreposição entre o limite superior da renda dos técnicos e o limite inferior da renda dos enfermeiros.

Tabela 2 - Rendimento médio real a preços constantes de 2018 de técnicos de enfermagem e enfermeiros, por anos selecionados (2003-2018).

\begin{tabular}{cccccccccc}
\hline Grupo & Limite & \multicolumn{2}{c}{$\mathbf{2 0 0 3}$} & \multicolumn{2}{c}{$\mathbf{2 0 0 8}$} & \multicolumn{2}{c}{$\mathbf{2 0 1 3}$} & \multicolumn{2}{c}{$\mathbf{2 0 1 8}$} \\
& & $\mathbf{R} \$$ & $\Delta \mathbf{2 0 0 3}$ & $\mathbf{R} \$$ & $\Delta \mathbf{2 0 0 3}$ & $\mathbf{R} \$$ & $\Delta \mathbf{2 0 0 3}$ & $\mathbf{R} \$$ & $\Delta \mathbf{2 0 0 3}$ \\
\hline \multirow{2}{*}{ Técnico } & Inferior & 961.70 & - & 1037.41 & \multirow{2}{*}{$7.87 \%$} & 1209.50 & \multirow{2}{*}{$25.77 \%$} & 1267.69 & \multirow{2}{*}{$31.82 \%$} \\
& Superior & 2885.11 & & 3112.22 & & 3628.49 & & 3803.08 & \\
\multirow{2}{*}{ Enfermeiro } & Inferior & 2757.02 & - & 2899.80 & \multirow{2}{*}{$5.18 \%$} & 2865.09 & \multirow{2}{*}{$3.92 \%$} & 2732.73 & $-0.88 \%$ \\
& Superior & 8271.05 & & 8699.40 & & 8595.28 & & 8198.18 & \\
\hline
\end{tabular}

Fonte: Autores, com base nos dados da RAIS.

Ocorreu, portanto, uma aproximação da renda da "classe média" dos dois grupos devido ao crescimento da renda dos técnicos e estagnação da renda dos enfermeiros. Uma análise mais precisa da redução da desigualdade, no caso dessa investigação, pelos Índices de Theil, apresentados nas Tabelas 3 e 4.

Tabela 3 - Evolução do L de Theil decomposto, por anos selecionados (2003-2018).

\begin{tabular}{cccccc}
\hline Ano & $\begin{array}{c}\text { Desigualdade } \\
\text { Intersetorial }\end{array}$ & $\begin{array}{c}\text { Desigualdade } \\
\text { Intrasetorial }\end{array}$ & Desigualdade Total & $\begin{array}{c}\text { Desigualdade } \\
\text { Intersetorial (\%) }\end{array}$ & $\begin{array}{c}\text { Desigualdade } \\
\text { Intrasetorial (\%) }\end{array}$ \\
\hline 2003 & 0.0723 & 0.1539 & 0.2263 & 31.98 & 68.02 \\
2008 & 0.0676 & 0.1529 & 0.2205 & 30.67 & 69.33 \\
2013 & 0.0625 & 0.1505 & 0.2131 & 29.34 & 70.66 \\
2018 & 0.0607 & 0.1345 & 0.1953 & 31.11 & 68.89 \\
\hline
\end{tabular}

Fonte: Autores, com base nos dados da RAIS.

Tabela 4 - Evolução do T de Theil decomposto, por anos selecionados (2003-2018).

\begin{tabular}{cccccc}
\hline Ano & $\begin{array}{c}\text { Desigualdade } \\
\text { Intersetorial }\end{array}$ & $\begin{array}{c}\text { Desigualdade } \\
\text { Intrasetorial }\end{array}$ & Desigualdade Total & $\begin{array}{c}\text { Desigualdade } \\
\text { Intersetorial (\%) }\end{array}$ & $\begin{array}{c}\text { Desigualdade } \\
\text { Intrasetorial (\%) }\end{array}$ \\
\hline 2003 & 0.0856 & 0.1532 & 0.2388 & 35.84 & 64.16 \\
2008 & 0.0775 & 0.1585 & 0.2360 & 32.85 & 67.15 \\
2013 & 0.0687 & 0.1637 & 0.2323 & 29.55 & 70.45 \\
2018 & 0.0648 & 0.1451 & 0.2099 & 30.87 & 69.13 \\
\hline
\end{tabular}

Fonte: Autores, com base nos dados da RAIS.

As tabelas mostram resultados similares para os dois Índices de Theil. A desigualdade total da enfermagem, considerando enfermeiros e técnicos como um só grupo, manteve-se próxima da estabilidade até 2013, sofrendo queda no último período estudado. De modo geral, a desigualdade pelo L de Theil diminuiu 13,7\% e pelo $\mathrm{T}$ de Theil 12,1\%.

A decomposição dos Índices de Theil aponta um peso maior da diminuição da desigualdade intersetorial entre os grupos, do que da intrasetorial, interna a cada um dos grupos, no resultado geral. Para o $\mathrm{L}$ de Theil, a desigualdade intrasetorial diminui $12,6 \%$ e a intersetorial 16,0\%. Para o T de Theil, a desigualdade intrasetorial diminuiu 5,3\% e a intersetorial 24,3\%.

Essa diferença pode ser, em parte, devido ao $\mathrm{L}$ de Theil ser mais sensível às oscilações da proporção de trabalhadores do grupo no total de trabalhadores, enquanto o $\mathrm{T}$ de Theil é mais sensível às variações da participação da renda de cada grupo no total da categoria enfermagem. Além disso, o L de Theil é mais sensível às variações na calda inferior da distribuição e, por outro lado, a sensibilidade do $\mathrm{T}$ de Theil independe do nível de renda. 
Esses resultados corroboram que ocorreu, entre 2003 e 2018, uma aproximação dos rendimentos do trabalho de enfermeiros e técnicos com carga horária semana de 40 a 44 horas. Dois dados importantes que não foram abordados nessa investigação de modo direto, mas que podem auxiliar na compreensão desses resultados são o aumento do salário mínimo e a modificação da composição do mercado de trabalho da enfermagem durante o período estudado.

Em valores corrigidos pelo IPCA, o salário mínimo aumentou, entre 2003 e 2018, de $\mathrm{R} \$ 551$,94 para $\mathrm{R} \$ 954,00$, um ganho real de $78,2 \%$. É provável que o efeito desse aumento foi mais relevante para o grupo com rendimentos menores, no caso, os técnicos.

No período estudado, a composição dos trabalhadores da enfermagem com vínculos de carga horária semanal de 40 a 44 horas modificou-se. Ocorreu uma expansão de 94,9\% na quantidade de vínculos entre 2003 (350.470) e 2018 (682.532). Também se observou uma modificação na proporção entre grupos. Para 2003, os técnicos perfaziam $84,4 \%$ e os enfermeiros $15,6 \%$. Para 2018 , os técnicos eram $71,4 \%$ e os enfermeiros $28,6 \%$.

\section{Discussão}

Este estudo apresenta achados relacionados à tendência salarial de profissionais de enfermagem, que revelam uma aproximação entre os salários dos profissionais de nível médio e os de nível superior, no período 2003-2018. Desse modo, observou-se que os salários dos técnicos mostram aumentos ascendentes no período de 2003-2013, década que o salário mínimo foi reajustado, com ganho real, acima da inflação. O mesmo não ocorreu para os enfermeiros, cujos vencimentos eram superiores aos valores do salário mínimo neste período, o que pode justificar a aproximação entre os salários e, até mesmo, a discreta redução nos salários dos enfermeiros nos anos subsequentes.

Possivelmente, essa aproximação deveu-se a um aumento da renda dos técnicos frente à estabilidade do valor recebido pelos enfermeiros. Observa-se que a desigualdade de renda da enfermagem diminuiu, no período estudado, principalmente à custa de redução de renda entre enfermeiros e técnicos. Ocorreu, portanto, uma aproximação da renda da "classe média" dos dois grupos devido ao crescimento da renda dos técnicos e estagnação da renda dos enfermeiros.

O mercado de trabalho do setor saúde apresenta assimetrias de salários entre as profissões (Yeager \& Leider, 2019), flexibilidades nas relações de trabalho e crescente feminilização (Madalozzo \& Artes, 2017). No caso específico da enfermagem, a categoria convive com distorções estruturais significativas quando comparados com salários auferidos por outras profissões (Ariste \& Béjaoui, 2015; Schenone, Rasero \& Cavaliere, 2018).

No complexo sistema de saúde brasileiro, Silva \& Machado (2019) alertam que, do mesmo modo que o SUS é subfinanciado desde sua criação, os profissionais de Enfermagem recebem baixa remuneração. Em determinadas regiões, tem sido recorrente o profissional acumular o exercício de atividades externas ao setor saúde, para complementar o rendimento mensal.

Os avanços tecnológicos das últimas décadas têm exigido da Enfermagem um esforço consistente para obtenção de mais prestígio e uma grande habilidade em demonstrar sua capacidade resolutiva junto aos problemas de saúde da população (Yoo et al., 2016). No entanto, esses avanços também têm efeitos negativos, gerando concorrência, disputas jurisdicionais no campo da regulação profissional, colocando em risco conquistas e, até mesmo, perda de espaço no mercado de trabalho (Machado et al., 2019).

O controle burocrático gerencial implantado nas organizações de saúde tem sido apontado como sendo um sinal de novos tempos das formas de regulação do trabalho em saúde, o que denota o desequilíbrio da regulação do Estado entre as profissões. É fato que o sistema de saúde brasileiro não tem respondido a essas novas exigências, tanto por parte dos profissionais de saúde quanto dos usuários. Ambos sentem-se insatisfeitos com os resultados desses avanços e as formas como o estado tem respondido a isso. Se por um lado as tensões das profissões sobre o Estado para o alcance de sua autonomia e 
controle de mercado são reconhecidamente legítimas, por outro, o Estado deve buscar promover um equilíbrio entre estes, de forma a garantir os princípios e diretrizes constitucionais para a saúde e tecer políticas de saúde para os seus trabalhadores (Machado et al., 2019; Silva et al., 2020).

Um dos importantes mecanismos na gestão do trabalho seria a estruturação do plano de carreiras, cargos e salários no sistema de saúde brasileiro. Isso propiciaria melhorias na gestão, com qualificação das práticas ofertadas nos serviços, a partir da valorização dos profissionais discutida e implementada de forma coletiva (Vieira et al., 2017).

Além disso, constata-se que os empregos assalariados que mais cresceram foram os dos trabalhos informais, cuja participação relativa na População Economicamente Ativa (PEA) ocupada passou de 14\%, em 1980, para quase 20\%, em 2018. A contrapartida disso foi o decréscimo do peso relativo do emprego formal de $78,3 \%$ do total dos assalariados para 70,4\% no mesmo período (Lima \& Costa, 2016; Pochmann, 2020).

Outra característica estrutural do mercado de trabalho no Brasil são os baixos salários. No ano de 2016, por exemplo, quase $71 \%$ das ocupações no Brasil recebiam até dois salários mínimos mensais, enquanto em 1986 eram de 68,1\%. Ou seja, em três décadas houve ampliação na proporção das ocupações que recebem até dois salários mínimos no total dos trabalhadores brasileiros. Em compensação, o segmento das ocupações com rendimentos intermediários, entre 2,1 a 5 salários mínimos mensais, decresceu em 3,6\% em relação ao total dos trabalhadores, pois decaiu de $22 \%$ para 21,2\%, entre 1986 e 2018. Também o segmento de maior rendimento, acima de cinco salários mínimos mensais, diminuiu em $19,2 \%$ a sua participação relativa no total das ocupações, passando de 9,9\% para 8\% no mesmo período de tempo (Pochmann, 2020).

\section{Conclusão}

De modo geral, a remuneração de enfermeiros e técnicos tem se tornado mais próxima nos últimos 15 anos devido à estagnação do valor recebido pelos enfermeiros e a um aumento do valor real recebido pelos técnicos, que acompanhou o salário mínimo. A situação das duas categorias profissionais é insatisfatória, pois frente ao desgaste físico e psicossocial originado pelo trabalho, muitas vezes em condições precárias, adicionam-se as dificuldades de sobrevivência com uma renda baixa. Os resultados dessa situação podem ser materializados na diminuição da qualidade do atendimento prestado por essa categoria profissional, dadas as suas condições precárias de trabalho.

Como recomendação, aponta-se a necessidade de estabelecimento de políticas públicas de valorização da categoria, incluindo a regulamentação de jornada de trabalho com carga horária específica e piso salarial nacional.

É relevante afirmar que este estudo apresenta limitações. A principal delas é ser baseada em dados secundários coletados para outra finalidade, portanto, é possível que existam inconsistências nos bancos de dados não identificadas pelos autores. Uma limitação adicional é a organização dos dados na RAIS por vínculo e não por trabalhador, o que impossibilita o acesso direto ao valor da remuneração total de cada trabalhador. Também, não é possível identificar rendimento adicional obtido pelos profissionais ao buscarem profissões complementares, como a de professor ou administrador. Não foram exploradas as diferenças regionais ou por sexo, que podem ter um papel importante nesse cenário.

\section{Referências}

Ariste, R., \& Béjaoui, A. (2015). Estimating Nursing Wage Bill in Canada and Breaking Down the Growth Rate: 2000 to 2010. Healthc Policy, 10 (4), 48-60. COFEN. (2019). Conselho Federal de Enfermagem. Enfermagem em números. http://www.cofen.gov.br/enfermagem-em-numeros.

Guerrero, J., \& Pulido, G. (2010). Trabajo, salud y regimén contractual em personal de enfermería: um enfoque psicosocial. Avances en Enfermería, 28(2), $111-122$.

Haughton, J. Inequality Measures. In: Haughton, J., \& Shahidur, R.K. (2009). Handbook on Poverty and Inequality. Washington: The World Bank. 
Research, Society and Development, v. 10, n. 3, e54210313569, 2021

(CC BY 4.0) | ISSN 2525-3409 | DOI: http://dx.doi.org/10.33448/rsd-v10i3.13569

Lima, T. B., \& Costa, M. S. (2016). Trabalho informal: uma revisão sistemática da literatura brasileira na área de Administração entre 2004 e 2013. Cadernos EBAPE.BR, 14 (2), 310-324.

Machado, M. H., Koster, I., Aguiar Filho, W., Wermelinger, M. C. M. W., Freire, N. P., \& Pereira, E. J. (2019). Mercado de trabalho e processos regulatórios - a Enfermagem no Brasil. Ciência \& Saúde Coletiva, 25 (1), 101-112.

Madalozzo, R., \& Artes, R. (2017). Escolhas profissionais e impactos no diferencial salarial entre homens e mulheres. Cadernos de Pesquisa, 47(163),202-21.

Melo, C. M. M., Carvalho, C. A., Silva, L. A., Leal, J. A. L., Santos, T. A., \& Santos, H. S. (2016). Força de trabalho da enfermeira em serviços estaduais com gestão direta: Revelando a precarização. Esc. Anna Nery, 20(3), e20160067.

Pereira, A. S. et al. (2018). Metodologia da pesquisa científica. UFSM.

Pew Research Center. (2015). The American Middle Class Is Losing Ground: No longer the majority and falling behind financially. 2015. https://www.pewsocialtrends.org/2015/12/09/the-american-middle-class-is-losing-ground/.

Pochmann, M. (2020). Tendências estruturais do mundo do trabalho no Brasil. Ciência \& Saúde Coletiva, 25(1), 89-99.

Schenone, D., Rasero, L., \& Cavaliere, B. (2018). Fee payment system for nursing performance: an operational proposal through the use of I.C.A. (Indexes of Complexity of Assistance) methodology. Acta Biomedica for Health Professions, 89(Suppl 6), 87-96.

Silva, M. C. N., \& Machado, M. H. (2019). Sistema de Saúde e Trabalho: desafios para a Enfermagem no Brasil. Ciência \& Saúde Coletiva, 25(1): 7-13.

Silva, R. M., Vieira, L. J. E. S., Garcia-Filho, C., Bezerra, I. C., Cavalcante, N. A., Borba Netto, F. C., \& Aguiar, F. A. R. (2020). Precarização do mercado de trabalho de auxiliares e técnicos de Enfermagem no Ceará, Brasil. Ciência \& Saúde Coletiva, 25 (1), 135-145.

Spetz, J. (2016). The Nursing Profession, Diversity, and Wages. Health Services Research, 51 (2), 505-510.

Vieira, S. P., Pierantoni, C. R., Magnago, C., França, T., \& Miranda, R. G. (2017). Planos de carreira, cargos e salários no âmbito do Sistema Único de Saúde: além dos limites e testando possibilidades. Saúde em Debate, 41(112), 110-121.

Yeager, V. A., \& Leider, J. P. (2019). The Role of Salary in Recruiting Employees in State and Local Governmental Public Health: PH WINS 2017. American Journal of Public Health, 109 (5), 683-685.

Yoo, B. K., Kim, M., Lin, T.C., Sasaki, T., Ward, D., \& Spetz, J. (2016). The effect of prior healthcare employment on the wages of registered nurses. BMC Health Services Research, 16(1), e412. 